\title{
A Facile Surface Treatment Utilizing Binary Mixtures of Ammonium Salts and Polar Solvents for Multiply Enhancing Thermoelectric PEDOT: PSS Films
}

\author{
Tsung-Che Tsai, Tsung-Han Chen, Hsiu-Cheng Chang, Chun-Hua Chen, Yi-Chia Huang, \\ Wha-Tzong Whang
}

Department of Materials Science and Engineering, National Chiao Tung University, Hsin-Chu, Taiwan, 30010, Republic of China Correspondence to: W.-T. Whang (E-mail: wtwhang@mail.nctu.edu.tw) or C.-H. Chen (E-mail: chunhuachen@mail.nctu.edu.tw)

Received 19 May 2014; accepted 13 August 2014; published online 25 September 2014

DOI: $10.1002 /$ pola.27402

KEYWORDS: conducting polymer; doping; seebeck; surface treatment; thermoelectric

INTRODUCTION Poly(3,4ethylenedioxythiophene):poly(styre nesulfonate) (PEDOT:PSS) is a widely investigated ionic conducting polymer (ICP) in many research aspects and serves also as a promising thermoelectric polymer along with its potentially high and tunable electrical conductivity. $^{1-3}$ The effectiveness of a thermoelectric material is related to the dimensionless figure-of-merit (ZT) defined as $\sigma S^{2} T / \kappa$, where $\sigma$ is the electrical conductivity, $S$ is the Seebeck coefficient, $\kappa$ is the thermal conductivity, and $T$ is the absolute temperature. ${ }^{4}$ Ongoing research efforts on PEDOT:PSS are largely targeted at improving the electrical conductivity mainly due to less-effective strategies in modifying Seebeck coefficient and the intrinsically low thermal conductivity.

Among the numerous electrical-conductivity-improving techniques, surface treatment with chemical solvents is a special branch in contrast to the conventional solution blending approaches, since it can be directly and repeatedly performed on baked ICP films. ${ }^{5-10}$ We have previously demonstrated that by spin-coating solvents containing polar groups, such as 2-methoxyethanol (ME) and ethylene glycol, on the pristine PEDOT:PSS film can significantly enhance the electrical conductivity. ${ }^{10}$ Similar enhancement was found by repeatedly immersing the PEDOT:PSS film into ethylene glycol. ${ }^{5}$ Surface treatments specifically for increasing the Seebeck coefficient have less been reported although its tuning is also crucial as the electrical conductivity for thermoelectric applications. However, to date, only few few blending additives, e.g., ammonium formate $\left(\mathrm{HCOONH}_{4}, \mathrm{AF}\right)^{11}$ and urea $\left(\mathrm{CO}\left(\mathrm{NH}_{2}\right)_{2}\right),{ }^{12,13}$ have been proven to effectively enhancing the Seebeck coefficient of the pristine PEDOT:PSS film.
In this work, a novel composite recipe coupling binary mixtures of the ME solvent and ammonium salt, which has respectively proven as the electrical conductivity and Seebeck coefficient enhancers, was for the first time introduced for treating the surface of baked PEDOT:PSS films. Two potential ammonium salts, $\mathrm{AF}$ and ammonium nitrate $\left(\mathrm{NH}_{4} \mathrm{NO}_{3}, \mathrm{AN}\right)$, with various salt/ME ratios were applied for identifying the most effective recipe and the resulting thermoelectric properties.

Table 1 shows the thermoelectric properties of the pristine PEDOT:PSS films and those surface treated with single reagent, $\mathrm{ME}$ or the aqueous solution of $\mathrm{AF}\left(\mathrm{AF}_{\mathrm{aq}}\right)$. As can be seen, the ME treatment on the pristine PEDOT:PSS film actually leads to at least three orders improvement in the electrical conductivity. The Hall measurement confirmed that the improvement originates from the simultaneously increased carrier concentration and mobility. However, the Seebeck coefficient simultaneously decreases with the ME treatment. In contrast, $\mathrm{AF}_{\mathrm{aq}}$ only slightly improves the electrical conductivity mainly due to the slight decrease in the carrier concentration and the slight increase in the carrier mobility, but noticeably, it specifically enhances the Seebeck coefficient up to $\sim 20 \%\left(1.0 M \mathrm{AF}_{\mathrm{aq}}\right)$.

The electrical conductivity and Seebeck coefficient are both related with the carrier concentration as expressed ${ }^{14}$ :

$$
p=\sigma / e \mu_{\mathrm{H}}=N_{\mu} \exp \left(-\mathrm{eS} / k_{B}\right)
$$

where $p$ is the carrier concentration, $e$ is the unit charge, $\mu_{\mathrm{H}}$ is the hall mobility, $N_{\mu}$ is the effective density of states of transport level, and $k_{B}$ is the Boltzmann constant. It is thus understandable that reducing the carrier concentration is a 
TABLE 1 Thermoelectric Properties of the Pristine and Surface Treated PEDOT:PSS Films

\begin{tabular}{llllll}
\hline $\begin{array}{l}\text { Treatment } \\
\text { Reagent }\end{array}$ & $\begin{array}{l}\text { Seebeck Coeffi. } \\
\left(\mathrm{V} \mathrm{K}^{-1}\right)\end{array}$ & $\begin{array}{l}\text { Electrical Conduct. } \\
(\mathrm{S} \mathrm{cm})\end{array}$ & $\begin{array}{l}\text { Carrier Concen. } \\
\left(\mathrm{cm}^{-3}\right)\end{array}$ & $\begin{array}{l}\text { Carrier Mobility } \\
\left(\mathrm{cm}^{2} \mathrm{~V}^{-1} \mathrm{~s}^{-1}\right)\end{array}$ & $\begin{array}{l}\text { Power Factor } \\
\left(\mu \mathrm{W} \mathrm{m} \mathrm{m}^{-1} \mathrm{~K}^{-2}\right)\end{array}$ \\
None & 19.11 & 0.33 & $1.6 \times 10^{19}$ & 0.13 & 0.01 \\
$\mathrm{ME}$ & 15.01 & 757 & $2.0 \times 10^{20}$ & 37.15 & 17.06 \\
$\mathrm{AF}_{\mathrm{aq}}(0.1 M)$ & 21.06 & 7.83 & $1.3 \times 10^{19}$ & 3.75 & 0.35 \\
$\mathrm{AF}_{\mathrm{aq}}(0.5 M)$ & 21.54 & 1.49 & $2.6 \times 10^{18}$ & 3.53 & 0.07 \\
$\mathrm{AF}_{\mathrm{aq}}(1.0 M)$ & 23.34 & 0.95 & $2.1 \times 10^{18}$ & 2.83 & 0.05
\end{tabular}

practicable way to increase the Seebeck coefficient. According to Table 1, the AF solution plays unique roles not only on reducing the carrier concentration to increase the Seebeck coefficient, but more significantly, on enhancing the carrier mobility of the pristine PEDOT:PSS film to lead the slightly improved electrical conductivities. It is thus reasonable to conclude that, by utilizing a binary mixture of $\mathrm{AF}$ and ME, the carrier concentration and mobility of the PEDOT:PSS film are expected to be effectively tuned to have an enhanced ZT.

Figure 1 shows the Seebeck coefficient, the electrical conductivity and the resulting power factor of the ME/salt surfacetreated PEDOT:PSS films. It is clearly that when the ammonium salt, AF or AN, incorporates with ME, the Seebeck coefficient increases with the salt concentration whereas the electrical conductivity shows an opposite tendency, indicating the dominative role of the ammonium salts as mentioned above $\left(\mathrm{AF}_{\mathrm{aq}}\right)$. In contrast to the ammonium salts, the quantity of ME is relatively constant since ME serves as a solvent in the binary mixture. In other words, the ME induced enhancement in the electrical conductivity is approximately independent of the salt concentration and is thus insufficient to cover the lost carrier concentration caused by the increased ammonium salts to keep a high electrical conductivity as the single ME treatment. Despite the binary mixtures seem not to be more effective than the single reagent
(ME or $\mathrm{AF}_{\mathrm{aq}}$ ) in individually enhancing the Seebeck coefficient or electrical conductivity, the resulting power factors are still several orders higher than the pristine film and reaches a similar level of the ME treated one (Table 1).

As aforementioned, $\mathrm{ME}$ is rather crucial but insufficient in quantity for enhancing the electrical conductivity of the PEDOT:PSS films. To strengthen the ME effect, we performed a special two-step procedure in which pure ME treatment takes the lead prior to the ME/salt ones. In contrast to the single treatment with the binary mixtures, the ME pretreatment could provide additional amount of $\mathrm{ME}$ and reaction time with the PEDOT:PSS films for independently tuning the electrical conductivity. As displayed in Figure 1, with the ME pre-treatment, the power factors are significantly enhanced for all the specimens mainly due to the twice improved electrical conductivities. The result also implies that $\mathrm{ME}$ and ammonium salts play their respective roles in enhancing the electrical conductivity and Seebeck coefficient of the PEDOT:PSS films.

Figure 2 shows the AFM phase images of the pristine and surface treated PEDOT:PSS films. The lighter and darker regions represent PEDOT- and PSS-rich phases, respectively. The less contrastive blurred image shown in Figure 2(a) indicates that the PEDOT and PSS chains of the pristine film are uniformly blended. When the surface treatments are
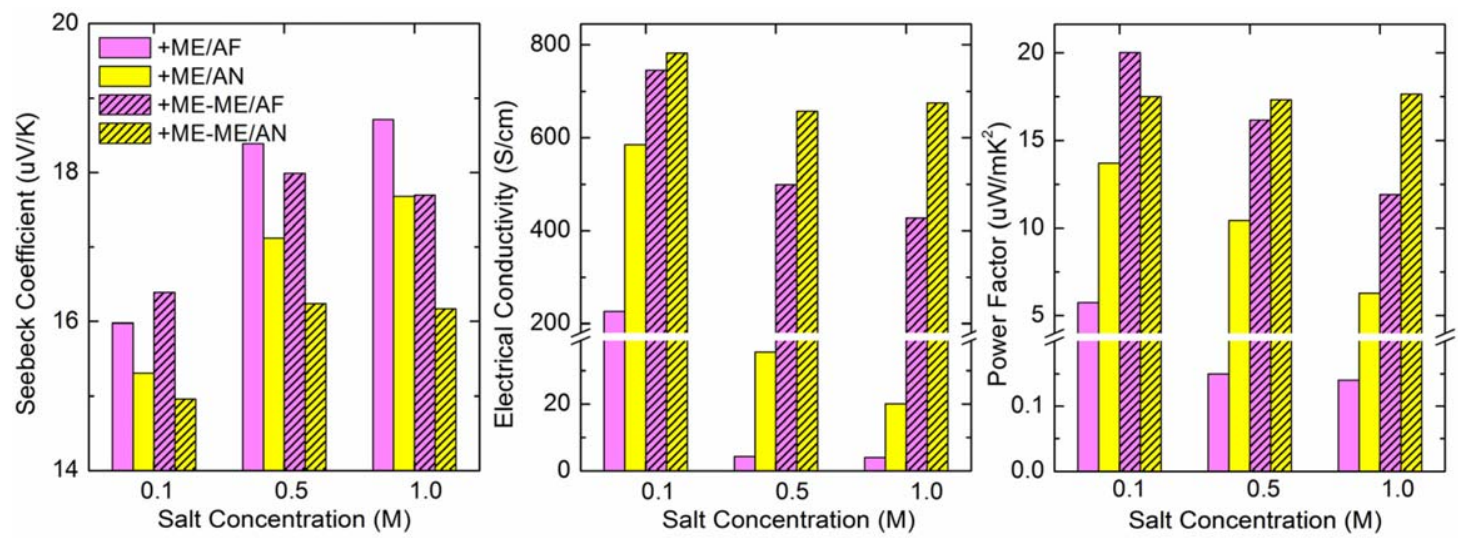

FIGURE 1 The Seebeck coefficients, electrical conductivities, and power factors of the ME/salt and ME-ME/salt (with ME pretreatment and then ME/salt) treated films. [Color figure can be viewed in the online issue, which is available at wileyonlinelibrary.com.] 

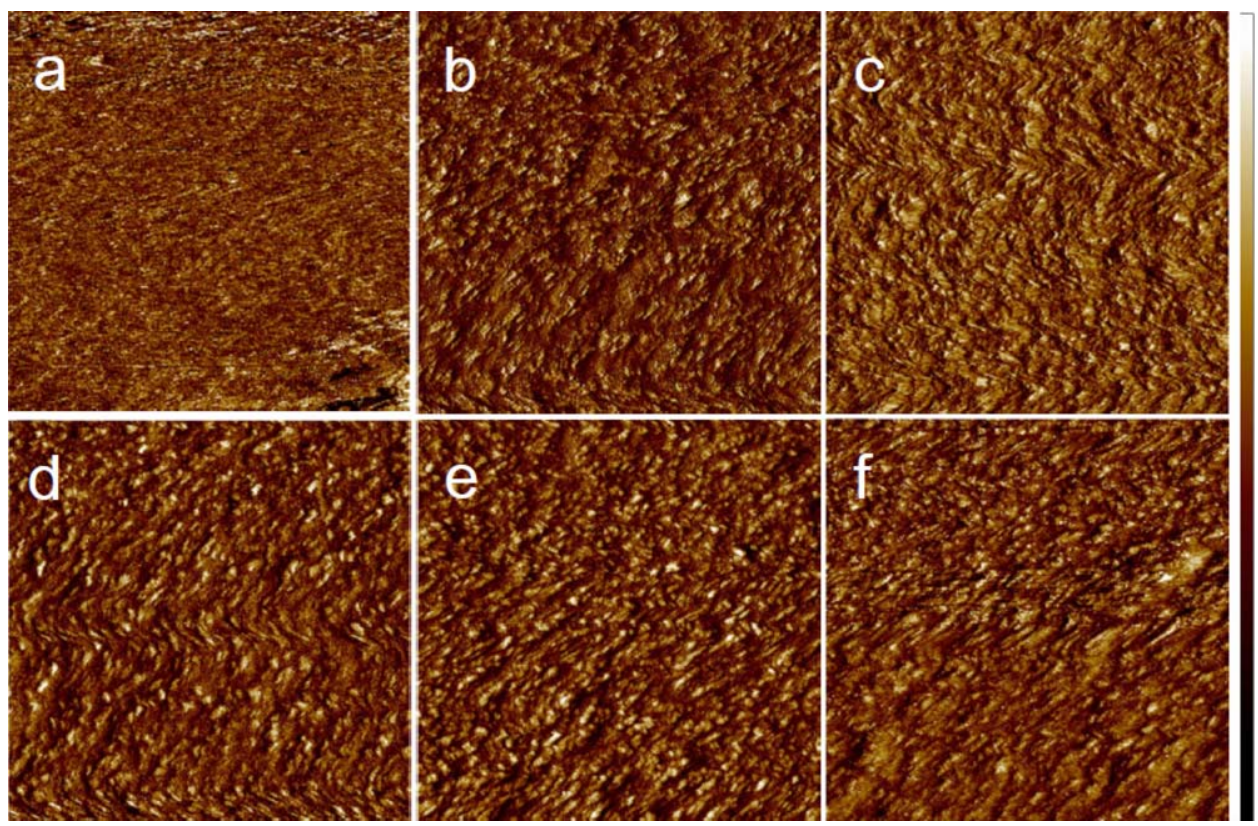

FIGURE 2 AFM phase images of (a) the pristine PEDOT:PSS film, and the (b) ME, (c) $A F_{a q}(0.1 M),(d) M E / A N(0.1 M),(e) M E / A F$ $(0.1 \mathrm{M})$, and (f) ME-ME/AF $(0.1 \mathrm{M})$ treated ones. The area scanned is $1 \times 1 \mu \mathrm{m}^{2}$ for each image. The color scale indicates a range of $\sim 800 \mathrm{mV}$. [Color figure can be viewed in the online issue, which is available at wileyonlinelibrary.com.]

performed, the much higher contrasts as observed in Figure 2 (b-f) strongly evidence the occurrence of the phase separation of PEDOT and PSS. This phenomenon originates from the great difference in the solubility of hydrophilic PSS and hydrophobic PEDOT with the polarity solvents, e.g., $\mathrm{H}_{2} \mathrm{O}$ or $\mathrm{ME}$, as reported previously. ${ }^{10}$ In addition, the charge screening effect, which would also lead to the phase separation and form a rather continuous network of PEDOT-rich phase, might occur with the presence of ME. Similar charge screening effect has also been reported for hydrolyzed hexafluoroacetone in PEDOT:PSS. ${ }^{15,16}$ The resulting aggregation of the PEDOT chains would specifically enhance the carrier mobility of the PEDOT:PSS films as found in Table 1. In comparison with the solvent of $\mathrm{ME}$, it was also found that the more polar molecule of $\mathrm{H}_{2} \mathrm{O}$ could dissolve and subsequently remove a greater amount of PSS to have a brighter AFM image [Fig. 2(c)].

Regarding the role of $\mathrm{AF}$ and $\mathrm{AN}$, it has been addressed that the ammonium could induce the desulfonation reaction on the sulfonic acid group of PSS. ${ }^{17,18}$ It is thus reasonable to believe that the ammonium obtained from the dissolved $\mathrm{AF}$ or $\mathrm{AN}$ in $\mathrm{H}_{2} \mathrm{O}$ or $\mathrm{ME}$ could also react with acid groups of PSS. In addition, a simple carrier exchange between the ammonium and $\mathrm{H}^{+}$of the PSS chains could reduce the active concentration of sulfonate on the PSS chains by forming ammonium sulfate. These two phenomena would lead to a lowering of the carrier concentration and thus the Seebeck coefficient of PEDOT:PSS.

However, with the copresence of $\mathrm{ME}$ and $\mathrm{AN}$ or $\mathrm{AF}$, the resulting phase distribution and ratio of PEDOT and PSS have a relative balance and are quite similar, well explaining their similar electrical conductivities. Figure 2(f) shows the AFM image of the two-step ME-ME/AF treated film. Although the amount of the dark PSS region is slightly lesser than Figure $2(\mathrm{~d}, \mathrm{e})$, the aggregated PEDOT region resulted from the pre-ME treatment is much larger, bringing the higher electrical conductivity.

The thermal conductivities were characterized following the ASTM D5470 protocol. It was found that the thermal conductivity is quite identical before and after the surface treatment, falling in the range of $0.195-0.213 \mathrm{~W} \mathrm{~m}^{-1} \mathrm{~K}^{-1}$. The optimized power factor and the resulted ZT of the ME/AF treated PEDOT:PSS films can achieve a rather high level of $\sim 20 \mu \mathrm{W} \mathrm{m}{ }^{-1} \mathrm{~K}^{-2}$ and 0.033 , respectively.

The innovative composite recipe coupling ammonium salts and ME was proposed and successfully demonstrated through the surface treatment method for improving the thermoelectric performance of the PEDOT:PSS films. The present remarkable enhancement in the resulting ZT is evidenced as the result of the phase separation brought by ME and the dedoping effect brought by $\mathrm{AF}$, which leads to the optimization of the electrical conductivity and Seebeck coefficient, respectively.

\section{EXPERIMENTAL}

The pristine film of PH 1000 is prepared by evenly spreading commercial PEDOT:PSS solution (Clevios PH 1000, Heraeus) onto the cleaned glass substrates $\left(2.5 \times 2.5 \mathrm{~cm}^{2}\right)$ with a subsequent baking process. Hundred microliter of pure ME or mixtures of ME and single ammonium salt, $\mathrm{AF}$ or $\mathrm{AN}$, was dropped onto the baked pristine PEDOT:PSS films and then 
the surface coated film was spun at $2000 \mathrm{rpm}$ and dried at $120^{\circ} \mathrm{C}$ for $1 \mathrm{~h}$. The electrical conductivity was acquired with an automated four-probe system and Hall measurement system (HMS-3000, ECOPIA). The Seebeck coefficients were recorded in situ with a maximum temperature gradient of $10^{\circ} \mathrm{C}$. Scanning electron microscopy (SEM, JSM-6500, JEOL) samples were broken in liquid nitrogen to avoid plastic deformation. Atomic force microscopy (AFM) phase images were captured in tapping mode (Veeco DiInnova system). Etched Si probes (resonance frequency $150 \mathrm{kHz}$, force constant $5 \mathrm{~N} \mathrm{~m}^{-1}$, tip radius of curvature $10 \mathrm{~nm}$ ) were used under ambient conditions. Typical scan rates during recording were $0.7-1$ line $s^{-1}$.

\section{ACKNOWLEDGMENTS}

The authors would like to acknowledge National Science Council, Republic of China, for the financial support through projects NSC 99-2221-E-009-010-MY3 and NSC 100-2221-E009-023-MY3.

\section{REFERENCES AND NOTES}

1 A. Elschner, S. Kirchmeyer, W. Lovenich, U. Merker, K. Reuter. PEDOT: Principles and Applications of an Intrinsically Conductive Polymer; CRC Press: Boca Raton, 2011.

2 K. C. Chang, M. S. Jeng, C. C. Yang, Y. W. Chou, S. K. Wu, M. A. Thomas, Y. Peng, J. Electron Mater. 2009, 38, 1182-1188. 3 J. Y. Kim, J. H. Jung, D. E. Lee, J. Joo, Synth. Met. 2002, 126, 311-316.
4 D. M. Rowe, Ed. CRC Handbook of Thermoelectrics. CRC Press: Boca Raton, 1995.

5 G. H. Kim, L. Shao, K. Zhang, K. P. Pipe, Nat. Mater. 2013, 12, 719-723.

6 J. Luo, D. Billep, T. Waechtler, T. Otto, M. Toader, O. Gordan, E. Sheremet, J. Martin, M. Hietschold, D. R. T. Zahn, T. Gessner, J. Mater. Chem. A 2013, 1, 7576-7583.

7 J. Ouyang, O. Xu, C. W. Chu, Y. Yang, G. Li, J. Shinar, Polymer 2004, 45, 8443-8450.

8 B. Peng, X. Guo, C. H. Cui, Y. P. Zou, C. Y. Pan, Y. F. Li, Appl. Phys. Lett. 2011, 98, 243308.

9 Y. Xia, K. Sun, J. Ouyang, Adv. Mater. 2012, 24, 2436-2440.

10 Y. S. Hsiao, W. T. Whang, C. P. Chen, Y. C. Chen, J. Mater. Chem. 2008, 18, 5948-5955.

11 T. C. Tsai, H. C. Chang, C. H. Chen, W. T. Whang, Org. Electron. 2011, 12, 2159-2164.

12 F. F. Kong, C. C. Liu, J. K. Xu, F. X. Jiang, B. Y. Lu, R. R. Yue, G. D. Liu, J. M. Wang, Chem. Phys. Lett. 2011, 28, 037201. 13 F. F. Kong, C. C. Liu, J. K. Xu, Y. Huang, J. M. Wang, Z. Sun, J. Electron. Mater. 2012, 41, 2431-2438.

14 B. Maennig, M. Pfeiffer, A. Nollau, X. Zhou, K. Leo, Phys. Rev. B 2001, 64, 195208.

15 Y. Xia, K. Sun, J. Ouyang, Energy Environ. Sci. 2012, 5, 5325-5332.

16 Y. Xia, J. Ouyang, ACS Appl. Mater. Interf. 2010, 2, 474-483. 17 S. A. Spanninga, D. C. Martin, Z. Chen, J. Phys. Chem. C 2009, 113, 5585-5592.

18 X. Crispin, S. Marciniak, W. Osikowicz, G. Zotti, A. W. Denier van der Gon, F. Louwet, M. Fahlman, L. Groenendaal, F. De Schryver, W. R. Salaneck, J. Polym. Sci. Part B: Polym. Phys. 2003, 41, 2561-2583. 